\title{
ISIS
}

\section{A Resource for Neutron Studies of Condensed Matter}

\section{J.L. Finney, Rutherford Appleton Laboratory}

(Chilton, Didcot, Oxon OX11 OQX)

In condensed matter structural studies, diffraction measurements using neutrons and $\mathrm{X}$-rays play a central role. These probes allow us to resolve detail over a range of length scales, from the interatomic to the longer distances that are characteristic of polymers and biomolecules.

With the advent of research reactors which culminated in the commissioning of the high flux reactor at the ILL Grenoble in the early 70 s, the scientific community began to exploit the specific advantages of the neutron. For example, a neutron of wavelength of about $1.5 \AA$ - as might be used for diffraction studies of atomic level structure - has an energy comparable with room temperature thermal fluctuations. Thus, in addition to elastic scattering measurements to probe condensed matter structure, inelastic measurements can be made in which the change in neutron energy on scattering reports back on the dynamics of the atoms of the sample. Furthermore, unlike $\mathrm{X}$-rays which are scattered by electrons, the neutron is scattered by the atomic nucleus. This has several consequences. First, the nucleus is a point scatterer; there is therefore no fall-off in the scattering factor (or length) with increasing scattering angle (or scattering vector $\mathbf{Q}$ ), an advantage in high resolution studies of structure. Second, different isotopes of the same element may scatter differently; thus, using isotope substitution, the scattering of chemically similar samples can be changed to great advantage. Finally, the neutron itself has a spin, which can interact also with electron spin; it is thus a powerful probe for the investigation of magnetic structure and excitations.

\section{Pulsed Neutrons: ISIS}

The impressive amount of neutron science performed over the past three decades has been done with neutrons produced by fission in the core of a reactor. The majority of experiments have required monochromatic incident beams, implying that a large fraction generally well above $90 \%$ - of the available neutrons were wasted. However, as the demand for more difficult experiments has increased, so has the need for more intense sources, but because of problems of heat removal from the reactor core, we may well be approaching the technological limits to power density.

An early appreciation of these problems was one factor which led to proposals in the 1950s to use pulsed accelerator sources, in which the neutrons are produced by the bombardment of a

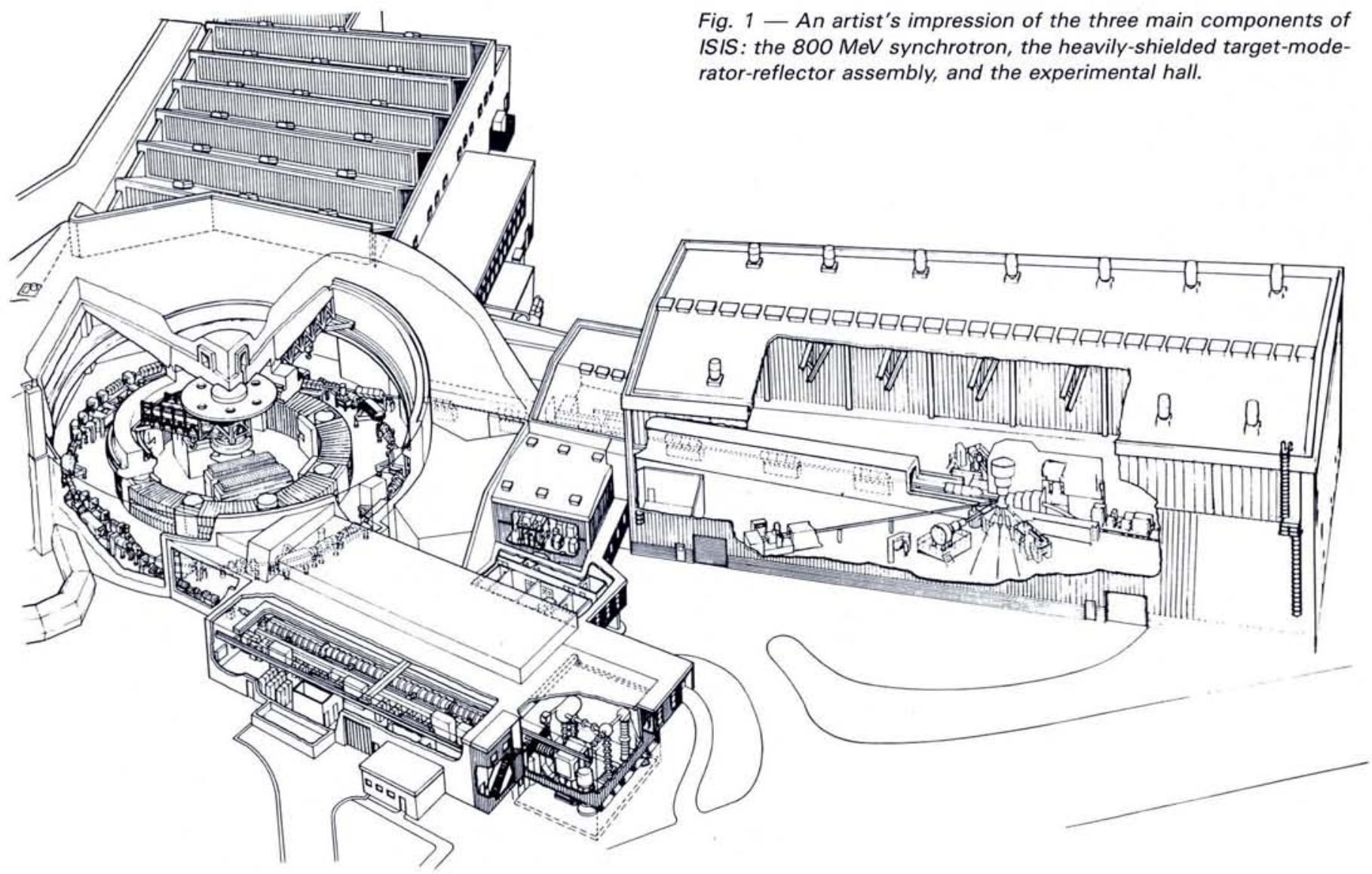

Fig. 1 - An artist's impression of the three main components of rator-reflector assembly, and the experimental hall. 


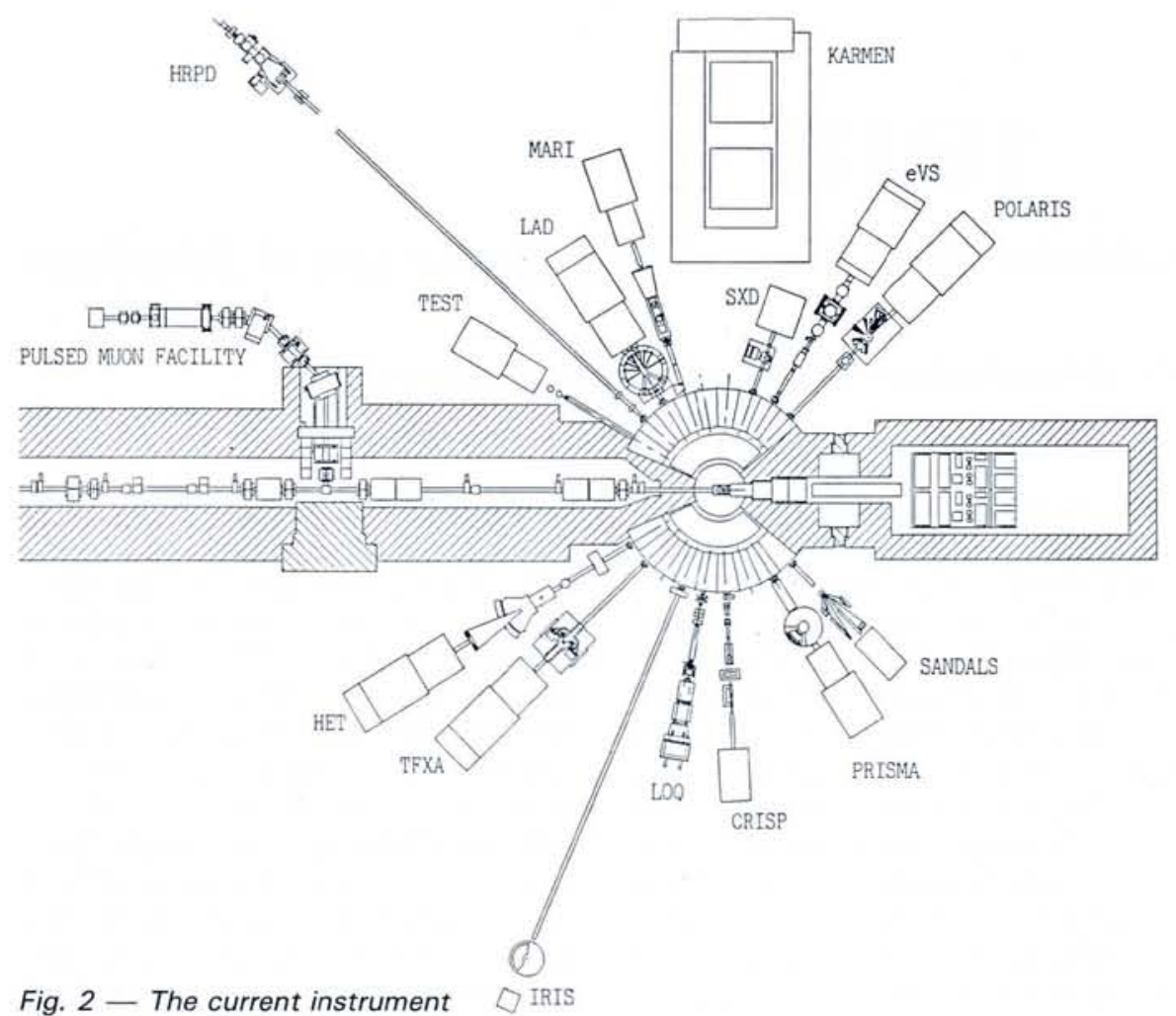

layout at the ISIS facility.

target by high energy electrons or protons. Proton spallation - in which the proton knocks bits off the heavy nuclei in the target - is the more efficient neutron producing process. Such a source - the Intense Pulsed Neutron Source IPNS - was commissioned at the Argonne National Laboratory in the USA in the early 1980s, and following a decision of the UK Science and Engineering Research Council made in the late 1970s, the pulsed neutron source ISIS was built at the Rutherford Appleton Laboratory, south of Oxford.

ISIS (Fig. 1) comprises three major parts: a fast cycling proton synchrotron (design current $200 \mu \mathrm{A}$ at $800 \mathrm{MeV}$ ), a ${ }^{238} \mathrm{U}$ spallation target feeding moderators at three different spectral temperatures, and a suite of neutron scattering instruments in the experimental hall. In addition, there is a muon spin rotation facility, and KARMEN, a collaborative neutrino experiment with the University of Karlsruhe. The first neutrons were produced in December 1984, and the condensed matter science programme commenced in August 1985 with five operational instruments. ISIS is currently running with high reliability at $100 \mu \mathrm{A}$ and $750 \mathrm{MeV}$. Integrated neutron production increased from $20 \mathrm{mAh}$ in 1986 , to $230 \mathrm{mAh}$ in 1988 , and projecting current performance, we expect to exceed 400 mAh in 1989. ISIS is now the world's most powerful pulsed neutron source. epithermal component allows uniquely high energy transfer in inelastic experiments, and high momentum transfer in elastic scattering, the significant cold neutron flux allied to the other advantages (especially resolution) make pulsed sources a uniquely powerful tool for cold neutron work. The conventional wisdom that pulsed sources are best for studies using high energy neutrons, while reactors should be used where cold neutrons are required, has already been demonstrated by ISIS to be incorrect. The compulsory use of time-offlight techniques to measure neutron wavelength, or energy, favours studies requiring a wide dynamic range in momentum or energy space. Moreover, as neutrons experiencing widely different momentum transfers are scattered at a particular angle we can work at fixed geometries, e.g. $90^{\circ}$. This facilitates greatly the use of special sample environments such as pressure cells and chemical reaction vessels.

\section{Instruments and Recent Science}

The instrumental suite at ISIS capitalises on these advantages of pulsed neutrons, to produce a scattering facility which is complementary to one that is reactor-based. The current instrumental complement (Fig. 2) includes elastic instruments for probing (crystalline and non-crystalline) structures, inelastic instruments for examining excitations and dynamics, plus a surface reflectometer for probing surface and interface structure.

It is not possible in an article of this length to give more than a taste of the science so far accomplished - or the potential for further exciting new areas. More details of recent work over the range of condensed matter science can be found in the ISIS annual reports ISIS 88 and ISIS 89, which can be obtained from the author.

\section{Crystallography}

The combination of wide spectral range, high resolution, and high brightness makes ISIS crystallography instruments unique. The high resolution powder diffractometer HRPD, with an unprecedented $d$-spacing resolution of $5 \times 10^{4}$, has extended the power of the powder diffraction technique considerably. Rietveld profile refinement of relatively complex structures can be carried out routinely, while $a b$ initio structure determination from powders - of great value when single crystals cannot be grown - is becoming increasingly straightforward. The uniquely high resolution also allows the study 
of hydrogeneous systems. Peak shape analysis has been transformed from something of a curiosity to a powerful, industrially-important technique, allowing detailed information to be obtained on, e.g. lattice strain, stacking faults and other defects as well as on anisotropic textures.

The quality of structural information available from HRPD is well illustrated by the detailed studies of the new "warm" superconductors. In the earliest work on the $35 \mathrm{~K}$ superconductor $\mathrm{La}_{1.85} \mathrm{Ba}_{15} \mathrm{CuO}_{4}$, subtle structural anomalies - missed by other workers in the field - were found around the superconducting transition. Detailed analysis of the diffraction peak shape indicated structural imperfections such as bulk microtwinning and oxygen stoichiometry gradients; this latter work raised serious questions concerning the methods of preparation of these potentially revolutionary materials.

The second arm of ISIS powder diffraction is POLARIS, a high intensity, medium resolution diffractometer, optimised for rapid measurements on standard-sized samples (and hence kinetic measurements during e.g. chemical reactions), or alternatively longer timescale measurements on very small (Xray size) samples. This latter possibility facilitates "raster scanning" measurements, in which structural variations across a sample (e.g. the residual stress distribution across a weld) can be measured at millimetre resolution. Both POLARIS and HRPD are equipped with $90^{\circ}$ detector banks to take advantage of the fixed geometry possibility for

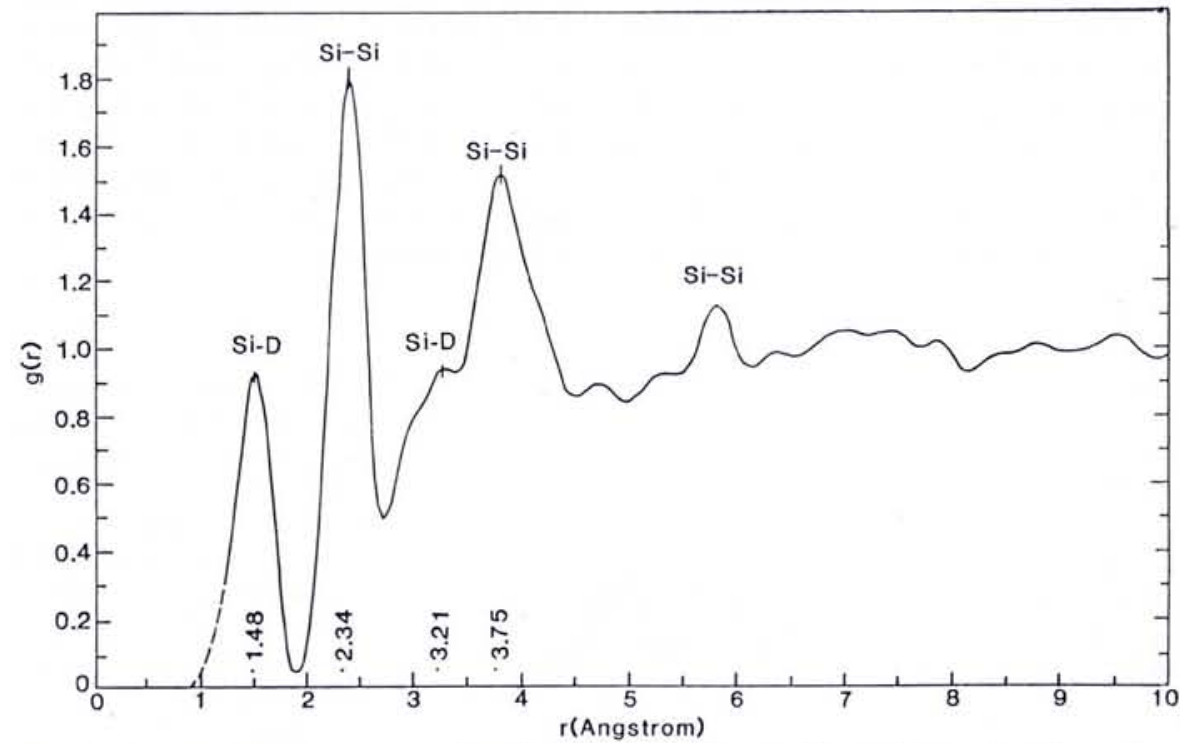

Fig. 3 - The pair correlation function of hydrogenated amorphous silicon, from data taken on LAD. An understanding of the structure of the system is essential if we are to understand its electronic properties.

(R. Bellissent, A. Menelle, W.S. Howells, A.C. Wright, T.M. Brunier, R.N. Sinclair and F. Jansen, Physica B, in press) high pressure work. POLARIS is, however, only a stepping stone to an even more powerful high intensity powder diffractometer currently under consideration. The consequent factor of about 50 reduction in either exposure times or sample sizes will open up qualitatively new scientific and engineering areas.

1989 has seen the coming on stream of our single crystal diffractometer SXD, particularly valuable for surveying reciprocal space at fixed geometry. The important region between the Bragg peaks can be examined to look for phase changes, with the appearance of superlattice or incommensurate peaks, and twinning. Diffuse elastic scattering can also give information on static disorder. Small single crystals can be studied with good signal to background ratios, and real-time crystallography, e.g. reaction kinetics of hydration, will be possible with single crystals. Plans are in hand for a longer flight path single cryscrystals to be studied with unit cell sides up to $100 \AA$.

\section{Liquids and Amorphous Materials}

Pulsed sources have major advantages over reactors for structural studies of liquids and amorphous materials. Profiting from the large epithermal neutron component, with the liquids and amorphous diffractometer, LAD, data can be taken over a wide range in $\mathbf{Q}$ (up to $50-100 \AA^{-1}$ ), resulting in increased real space resolution. $A$ wide range in $\mathbf{Q}$ values can be covered even at low angles, which allows us to minimise troublesome inelasticity (Placzek) cortal instrument designed to allow protein rections for samples containing light atoms - in particular hydrogen and deuterium. This is illustrated in a recent French study of the structure of the technologically important material amorphous silicon:hydrogen, see Fig. 3 . This ability to work at low scattering angle also facilitates the exploitation of $\mathrm{H}-\mathrm{D}$ substitution measurements to probe $\mathrm{H}-\mathrm{H}$ correlation functions in a variety of systems of both physical and chemical interest.

A second generation instrument, SANDALS, is now under construction which will allow a further reduction in kinematic effects, a greatly improved statistical quality in isotope substitution experiments, and an extension of measurements in the low $\mathbf{Q}$ region which is important in understanding interatomic potentials.

\section{Surface Studies}

CRISP is a powerful instrument for studying surfaces and interfacial phenomena. As the neutron refractive index of materials is - like X-rays - less than one, a beam incident on the surface at a small angle $\left(\theta<\theta_{\text {crit }}\right)$ will be totally externally reflected. Measurements on a thin film will give interference fringes beyond the region of total reflection; we can deduce information on the density of the layer, the layer thickness, and details of the nature of the air/film and film/substrate interfaces. CRISP has both an inclined beam for investigating liquid samples, and a polarised beam option to study surface magnetism.

The CRISP scientific programme ranges widely through surface chemistry (probing adsorption at simple and complex air-liquid, liquid-liquid, and liquidsolid interfaces), surface magnetism, and low dimensional structures. The programme on solid films has examined, for example, a variety of multilayers (e.g. GaAs/AlAs, $\mathrm{Pt} / \mathrm{C}$ ), hard carbon films, Langmuir-Blodgett films, and polymer-polymer interfaces. The study of surface magnetism is an area uniquely suited to neutron reflection, the CRISP programme here including magnetisation in ferromagnetic glasses and flux penetration in high $T_{c}$ superconductors. The power - and effectiveness - of this instrument to give useful information on real, relatively complex systems, is witnessed by the fact that around half the experiments on CRISP relate to industrial problems.

\section{Large Scale Structures}

Small angle scattering is a powerful tool used at all neutron sources to give 
information on large scale structures in materials science, polymer studies and biology. Pulsed source instruments such as LOQ at ISIS cover simultaneously a wide $\mathbf{Q}$ range allowing dynamical effects such as precipitation and defect growth to be followed with ease. Fig. 4 shows the results from an Italian experiment to measure the effects of clustering in $\mathrm{Al}-\mathrm{Li}$ alloys, which are extensively used in the aerospace industry. The performance of LOQ towards higher values of $\mathbf{Q}$ is particularly good, and this can be valuable when internal structural information is required. The current scientific thrust in small angle scattering at ISIS is to investigate more complex materials and systems, as well as more "traditional" samples under new and more exotic sample environments. The former class might include copolymers and polymer blends, films under stress, membranes, concentrated solutions and gels, and anisotropic particles. More complex sample environments include measurements under shear flow, magnetic fields and electric fields.

\section{Inelastic Scattering}

At ISIS, the study of the dynamics of condensed matter ranges in energy from microelectron volts to an electron volt. These excitations are probed using a suite of three inelastic spectrometers (HET, TFXA and IRIS) with two instruments at an advanced stage of commissioning (PRISMA and eVS). The MARI project (funded by Japan) will be completed later this year.

The high energy transfer spectrometer HET exploits the rich epithermal spectrum to investigate high energy transfer processes at low values of associated momentum transfer. The excellent resolution and low background have enabled HET to make significant contributions using neutrons with energies as low as $35 \mathrm{meV}$ and as high as $2000 \mathrm{meV}$.

Examples of recent experiments in the low energy region include electronic states in UOS, magnetic response of $\mathrm{NdFeO}_{3}$, excitation and spin dynamics of the heavy fermion system $\mathrm{CeCu}_{2} \mathrm{Si}_{2}$, and the modes of hydrogen in thulium hydride. HET has also been able to tackle some of the potentially very large number of problems in rare earths and actinides (e.g. heavy fermions, valence fluctuations). Even studies of excitations in single crystals, which involve detailed investigations of a limited and specific volume of $(Q, \varepsilon)$ space (not traditionally thought to be the forte of the time of flight technique) have been

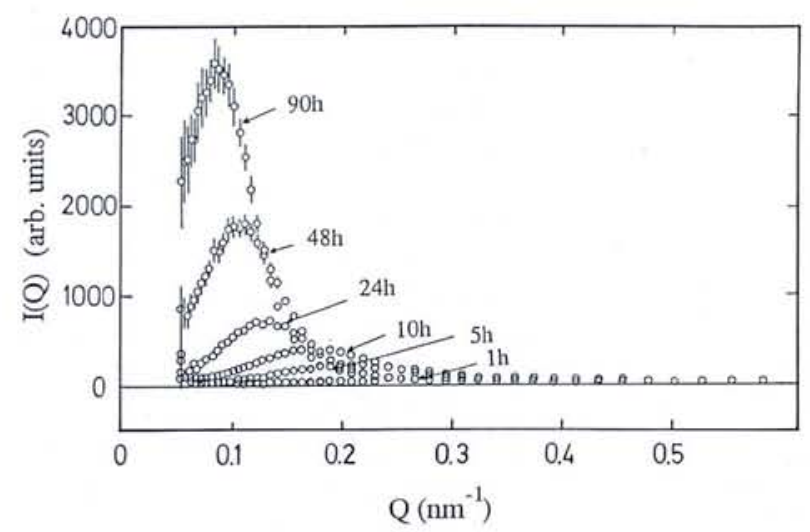

Fig. 4 - The growth of lithium precipitates in $\mathrm{Al}-\mathrm{Li}$ alloys from data taken on LOQ, over 1 to 90 hour annealling times. The data is being used to develop models of the kinetics of growth and the precipitate size distribution.

(S. Abis, R. Caciuffo, F. Carsughi, R. Coppola, R.K. Heenan, R. Osborn and $M$. Stefanon, Physica $\mathrm{B}$, in press.)

highly successful. An example is the measurement of the dispersion relation of spin waves in cobalt out to the zone boundary at around $300 \mathrm{meV}$.

The main thrust of the HET programme currently, however, is in magnetism. A new field, unique to HET, is intermultiplet spectroscopy of $4 f$ and $5 f$ electrons in metallic systems. In addition to observing the lowest level at 260 $\mathrm{meV}$ in metallic praseodymium and confirming that its form factor had significant non-dipolar terms, higher order transitions up to the ${ }^{3} \mathrm{H}_{4} \rightarrow{ }^{3} \mathrm{~F}_{4}$ at 810 meV were observed (Fig. 5). This measurement - almost a factor of three higher in energy than any magnetic excitation previously seen with neutrons - showed that in the metal there was significant screening by the conduction electrons of the Coulomb interaction between the $4 f$ electrons. These measurements are of particular interest in the actinides, where recent work on $\mathrm{UPd}_{3}$ has led to the first unambiguous determination of the valency of uranium in a metallic compound.

When MARI - the multiangle rotor instrument financed through an agreement with Japan - becomes operational later in 1989, a wide and continuous coverage of $(Q, \varepsilon)$ space will become available at high resolution. In addition to allowing more detailed stu-

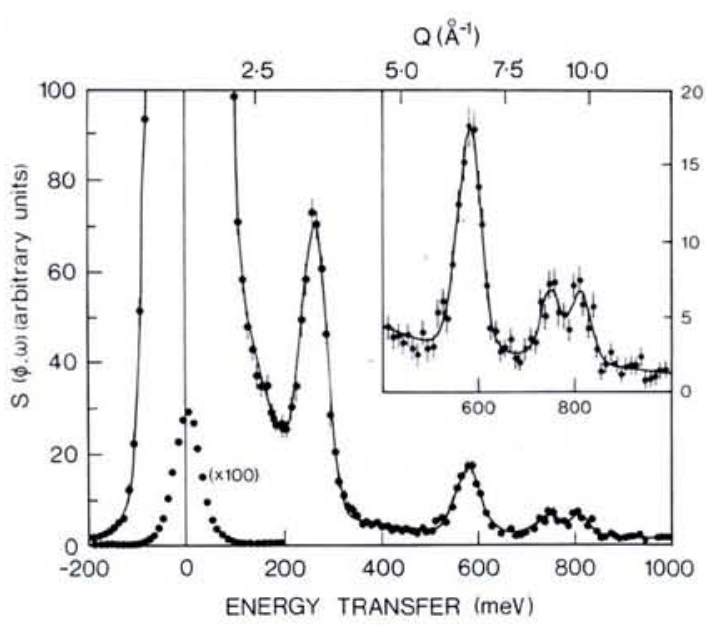

dies of magnetic and vibrational processes, it will be particularly useful for determining the dynamical structure factor $S(Q, \varepsilon)$ in liquids and amorphous solids.

The final ISIS instrument capable of studying high energy excitations is the electron volt spectrometer eVS. This is now being scheduled for studies of the momentum distribution of atoms in quantum systems. There is much theoretical interest in such studies, and recent experiments have looked at high $\mathbf{Q}$ scattering from mass 1 systems such as hydrogen atoms in double well potentials. Experiments on other systems will follow.

PRISMA - an instrument constructed by CNR in Italy through another bilateral agreement — is designed to measure coherent excitations in single crystals. It has already demonstrated (Fig. 6) its ability to measure simultaneously many loci in $(Q, \varepsilon)$ space, thus providing information complementary to that obtainable by triple axis spectroscopy on reactor sources, a technique which uses point-by-point scans. A second type of spectrometer designed to complement a triple axis instrument is ROTAX, which has one nonlinearly rotating crystal analyser. This is being developed on ISIS by the University of Würzburg. 


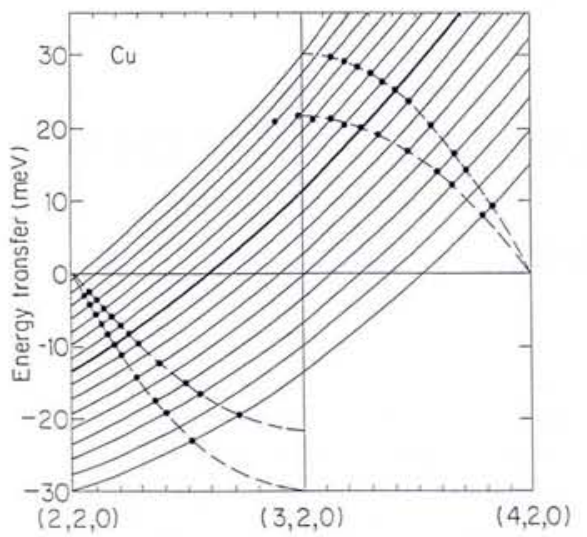

Fig. 6 - The phonon dispersion relation measured along the [100] direction in a single crystal of copper during early tests on $P R I S M A$. The solid lines are the loci in $(Q, \varepsilon)$ space scanned by each of the 16 detectors, while the dashed lines indicate the known dispersion relation.

The high resolution inelastic spectrometer IRIS operates in the cold neutrons range; it combines the advantages of high brightness, high resolution and spectral range to give detailed information on the dynamics of molecules. The good resolution at large energy transfers from very cold samples allow IRIS to make a unique contribution to quantum tunnelling studies in crystals, where a major interest is in studying the transition between quantum and classical regions. A recent example of the power of IRIS in such studies is the simultaneous observation by a German group of classical rotation and quantum tunnelling of the two (inequivalent) methane molecules in crystalline methane. Earlier measurements revealed intriguing behaviour of the quantum tunnelling levels of the methyl group in 4-methyl-pyridine $\mathrm{C}_{5} \mathrm{NH}_{4} \cdot \mathrm{CH}_{3}$ (Fig. 7).

The resolution and momentum transfer range of IRIS combine to make it a very effective machine to study the dynamics of rotational and translational diffusion. Such measurements, which were begun in earnest some 20 years ago with the advent of cold neutron sources on reactors, have always been hampered by the restriction in the $\mathbf{Q}$ range imposed by the large wavelength neutrons. IRIS promises to open up a new chapter in these studies. It is also leading to developments in quasielastic studies of more complex systems such as organic liquids and glasses, binary and ternary metallic alloy hybrides, the dynamics of biomolecules, and in multiphase systems.

Molecular spectroscopy is a field in which three ISIS instruments - HET, IRIS, and the time focussed crystal analyser spectrometer TFXA, form a formidable complementary group. The high resolution and wide energy transfer range available have helped us to improve our understanding of combination modes of internal and external vibrations in molecular systems, to study the effects of molecular recoil, and to probe molecular force fields. In the latter areas, TFXA has made notable contributions, and the resolution available is promising a revolution in molecular spectroscopy. Particularly exciting results have also been obtained in studies of real catalysts. An example is the identification of molecular hydrogen, rather than the expected atomic species, in the hydrodesulphurisation catalyst $\mathrm{MoS}_{2}$ supported on alumina. Fig. 8 shows the main evidence for this conclusion.

\section{SUMMARY}

Pulsed neutrons offer advantages of high incident energies, very high resolution in both time and space, and very low backgrounds which favour the development of new areas of neutron science and inject new life into old ones. ISIS instruments were designed specifically to capitalise on these advantages, and the results even at this early stage in the development of the facility have more than justified the expectations.

ISIS is however still very much a young facility, its neutron scattering programme being barely three years old, and its scheduled instrument complement well under half the potential of the current target station. Despite its youth, it has already established itself as the world's premier, as well as the most powerful, spallation neutron source. It is already producing an impressive amount of new science through experiments undertaken by scientists from many countries. Currently European bilateral agreements have either been signed or are under negotiation with Italy, France, the Netherlands, Sweden and the University of Würzburg. In ISIS, the UK and its partners have at their disposal a unique scientific and industrial research facility, capable of pushing back frontiers across most scientific disciplines - chemistry, biology, materials science as well as physics - and several aspects of engineering. It is essentially complementary to the reactorbased facilities available at the ILL, and the various domestic reactors. The potential for further growth - both with respect to increased international collaboration and its science - is really immense.
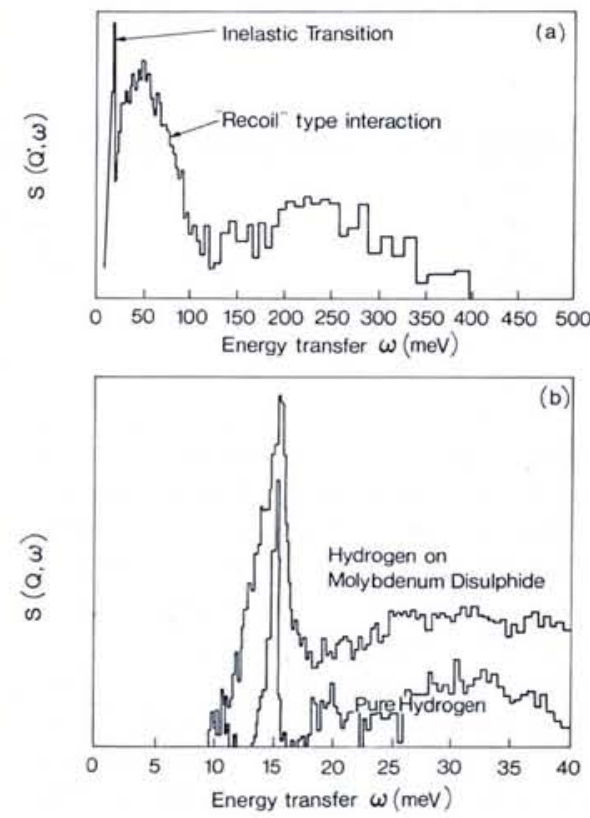

Fig. 7 - The presence of four levels in the previously unresolved tunnelling transition of 4-methyl pyridine, confirmed by both maximum entropy decomposition and Gaussian synthesis. The multiplicity of the spectrum derives from the coupling of the molecules to its surrounding, but the precise nature of this coupling remains controversial.

(F. Fillaux, C.J. Carlile, S. Clough, A.J. Horsewill and A. Smith, Chem. Phys., in press)

Fig. 8-TFXA measurements of the spectra of hydrogen on $\mathrm{MoS}_{2}$, a hydro-desulphurisation catalyst. The presence of the characteristic ortho $\rightarrow$ para conversion mode at $14.8 \mathrm{meV}$, and a higher energy recoil pattern consistent with that of a mass 2 particle, confirm that the hydrogen resides on the surface in molecular - not atomic - form.

(P.N. Jones, E. Knözinger, W. Langel, R.B. Moyes and J. Tomkinson, Surface Science 207 (1988) 159

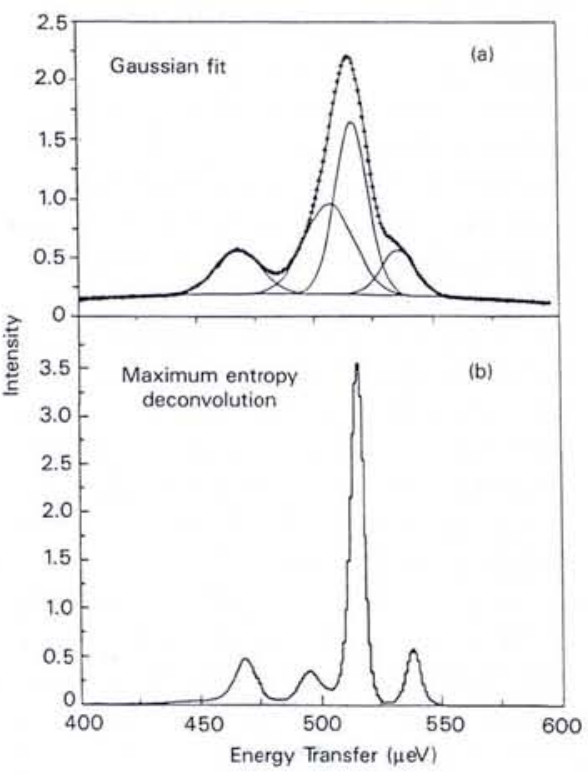

